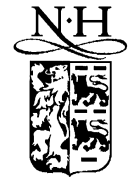

ELSEVIER

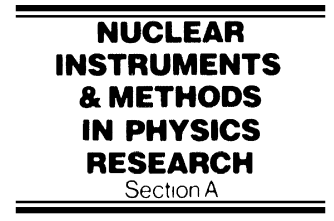

www.elsevier.nl/locate/nima

\title{
Glass capillary X-ray lens: fabrication technique and ray tracing calculations
}

\author{
Yu.I. Dudchik $^{\mathrm{a}, *}$, N.N. Kolchevsky ${ }^{\mathrm{a}}$, F.F. Komarov ${ }^{\mathrm{a}}$, Y. Kohmura ${ }^{\mathrm{b}}$, \\ M. Awaji ${ }^{\mathrm{c}}$, Y. Suzuki ${ }^{\mathrm{c}}$, T. Ishikava ${ }^{\mathrm{b}, \mathrm{c}}$ \\ anstitute of Applied Physics Problems, Kurchatova 7, Minsk 220064, Belarus \\ ${ }^{\mathrm{b}}$ Harima Institute, The Institute of Physical and Chemical Research (RIKEN), Mikazuki, Sayo-gun, Hyogo 679-5143, Japan \\ 'Japan Synchrotron Radiation Research Institute (JASRI), Mihara 323-3, Mikazuki Sayou-gun, Hyogo 6795198, Japan
}

Received 26 November 1999; received in revised form 6 April 2000; accepted 13 April 2000

\begin{abstract}
A new method for producing a compound refractive X-ray lens is described. The lens is designed as glass capillary filled by a set of concave individual lenses. The method is appropriate for the preparation of $10-1000$ spherical lenses in a glass capillary with a diameter of 0.1-1 mm. Lens aberrations are considered and simple formulas for spot size are derived. Ray-tracing simulations on the focusing experiments using $18 \mathrm{keV} \mathrm{X-rays} \mathrm{are} \mathrm{reported.} \mathrm{(C)} 2000$ Elsevier Science B.V. All rights reserved.
\end{abstract}

PACS: 07.85.Qe; $07.85 \mathrm{Yk} ; 41.50 .+\mathrm{h} ; 61.80 . \mathrm{Cb}$

Keywords: X-ray optics; Refractive lenses; Glass capillaries; Aberrations

\section{Introduction}

The appearance of the third-generation synchrotron radiation sources, such as ESRF, SPring-8 and APS has caused a rebirth of interest in the X-ray refractive optics. Since the refractive index of the materials for X-rays is less than unity, the converging X-ray beam may be obtained by using a concave lens instead of convex one for visual light. Calculations have shown that the focal length of the $\mathrm{X}$-ray lens is $50-100 \mathrm{~m} \mathrm{[1]}$. Attempts to reduce

*Corresponding author. Fax: + 172-780-417.

E-mail address:dudchik@pfp.bsu.unibel.by (Yu.I. Dudchik). the focal length result in a novel lens recently proposed by Snigirev et al. [2] which was named as a compound refractive lens for X-rays. The lens consists of a linear array of many individual concave lenses made from low- $Z$ material and at first was produced by drilling a number of holes in a block of aluminum alloy. The space between the holes played the role of an X-ray lens. The number of the holes was 30 and its diameter was $0.6 \mathrm{~mm}$. The lens was used for linear focusing of $14 \mathrm{keV}$ $\mathrm{X}$-rays into a line of several microns in width [2].

Two-dimensional focusing of $30 \mathrm{keV}$ synchrotron [3] and undulator [4] radiation also was achieved with the use of a lens in crossed geometry. Compound X-ray lenses (linear and crossed linear) made of beryllium, boron nitride, pyrographite, 
plexiglas, polycarbonate, polyoxymethylene and Vespel were tested at ESRF in terms of the focal spot size and gain and background [5]. The photon energies varied between 9 and $27 \mathrm{keV}$. The achieved gain reached 13 and this value is many times less than the maximum expected gain of 5000. As was noted in Ref. [5], deviations from the cylindrical shape of the holes, surface roughness, and smallangle scattering are the main reasons for this loss.

The studies on X-ray lenses at ESRF resulted in $\mathrm{X}$-ray microscope [6]. The main part of this microscope is a parabolic X-ray lens made from polycrystalline aluminum by a pressing technique. Magnification upto 50 with a resolving power of $0.3 \mu \mathrm{m}$ was achieved by using $23.5 \mathrm{keV} \mathrm{X}$-ray beam.

Recent experiments at SPring-8 [7,8] have shown a suitability of the "bubble" lens to focus 17.2 and $19 \mathrm{keV}$ undulator radiation. The lens was produced by placing gas bubbles on the surface of an adhesive liquid. The liquid located between the two air bubbles acted as the individual concave lens.

The lenses used in Refs. [2-8] were $0.5-2.8 \mathrm{~mm}$ in diameter and suitable for focusing of $14-30 \mathrm{keV}$ $\mathrm{X}$-ray beam at a distance of 1 to $2 \mathrm{~m}$. To reduce the focal length of the lens up to $10 \mathrm{~cm}$ the lens diameter should be less than some hundreds of microns. Such microlenses are difficult to realize by drilling or pressing technique but they may be formed in a glass microcapillary as proposed in Ref. [9]. The microcapillary X-ray lens was designed as a set of glue concave spherical lenses placed in a glass microcapillary [9]. The lenses were recently tested at SPring-8 undulator beamline using $18 \mathrm{keV}$ $\mathrm{X}$-rays [10]. It was found [10] that the lenses may be useful not only for focusing of X-rays but also for imaging applications: the gold mesh (with wire diameter of $5.6 \mu \mathrm{m}$ with pitch of $16.7 \mu \mathrm{m}$ ) was placed before the lens and was observed with a magnification factor of around 3 at the focal plane using $17 \mathrm{keV}$ X-rays. Two types of microcapillary lenses were used for these experiments [10]. One lens was fabricated using 70 glue microlenses in a glass capillary with a diameter of $0.2 \mathrm{~mm}$, while another one was fabricated using 185 glycerin microlenses inside a glass capillary with a diameter of $0.86 \mathrm{~mm}$.

The main disadvantage of the microcapillary lens described in Ref. [9] is a large thickness of the individual lens which approximately equals the lens diameter. As a consequence, the expected transparency of a shot focal length lens is too little to be practically useful.

The objective of this paper is to describe a new method for producing an array of concave liquid or polymer spherical lenses in a glass capillary with a diameter of $0.1-1 \mathrm{~mm}$. Spherical aberrations of the lens are considered and ray-tracing simulations of the X-ray lens used for the focusing experiments at SPring-8 [10] using the $18 \mathrm{keV} \mathrm{X}$-rays are reported.

\section{Glass capillary lens fabrication}

The main idea of the lens design is based on a well known fact that the surface of water in a glass tube will be curved and this curvature is concave. So a drop of water or a glue to be placed in a glass capillary forms concave lens due to surface tension forces. The surface of the drop can be approximated by a sphere when the capillary radius is smaller than 1-2 $\mathrm{mm}$. The radius $R$ of the surface of the drop and that of the capillary $R_{\mathrm{c}}$ are related by the following equation:

$R=R_{\mathrm{c}} / \cos \Theta$

where $\Theta$ is the contact angle. The contact angle between glass and water is $0^{\circ}$, and the one between glass and glycerine is about $0^{\circ}$ as well.

For the compound X-ray lens to operate successfully, individual lenses should be done of low- $Z$ materials [2]. Different liquids, such as water or glycerine, and glues may be used as the materials for the lens production. As for glues, most of the commercially available ones are polymers with a density between 0.9 and $1.3 \mathrm{~g} / \mathrm{cm}^{3}$ and the main chemical components of which are carbon and hydrogen.

The lenses used in Ref. [10] for focusing X-rays, were produced by two different methods. The first method (method 1) is the same as the one described in Ref. [9]. Some more details of the method are described in this paper. The method is appropriate only for producing concave lenses in glass microcapillaries of $0.05-0.3 \mathrm{~mm}$ in diameter and this limitation is caused by the significant absorption by 


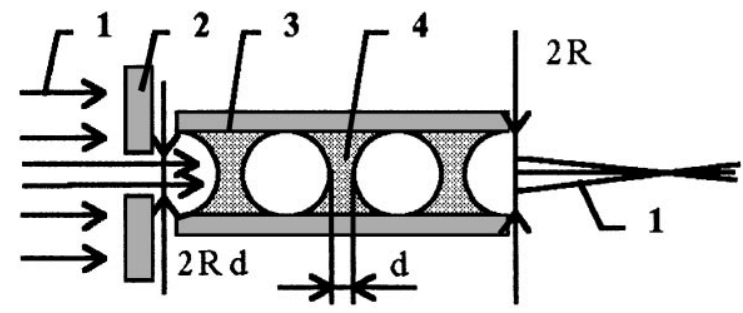

Fig. 1. Schematic view of the capillary lens. $1-\mathrm{X}$ ray beam; 2 - diaphragm; 3 - capillary; 4 - glue lens.

the finite thickness $d$ of the microlenses (Fig. 1). We have found empirically that a thin film of liquid formed inside the glass capillary collapses spontaneously and forms a set of drops. The drops have the form of the concave lens. The inner surface of the capillary is covered by the film in the following way. At first the capillary is filled by the liquid or glue and then connected to a cylinder with compressed air. The pressure of the air forces the glue out and forms a thin film of glue on the inner surface of the capillary. The air supply is cut off at the instant the glue leaves the capillary. It needs some minutes for the film to collapse. The number of the drops is limited only by the capillary length and, for example, it is possible to form 1000 lenses in one capillary. The empirical relationship between the individual lens thickness $d$ and the capillary radius $R$ is described by the following linear equation [9]:

$d=k R+d_{0}$

where $k=3$ and $d_{0}=-60 \mu \mathrm{m}$. We have found that the equation is valid for $R$ between 50 and $500 \mu \mathrm{m}$ and for different kinds of liquids (water, glycerine, glues).

The disadvantage of the method 1 is the large lens thickness for capillaries of $0.3-1 \mathrm{~mm}$ in diameter. This has been completely overcome by the second method (method 2). This method is more complicated than the first one and is suitable for obtaining concave lenses with a diameter of 0.1-1 mm. Two glass capillaries are used for manufacturing the lenses (Fig. 2). Capillary-1 is pre-filled by organic liquid and is placed under the light microscope. Capillary-2 is used as a "long glass injector needle" for putting air bubbles into capil-

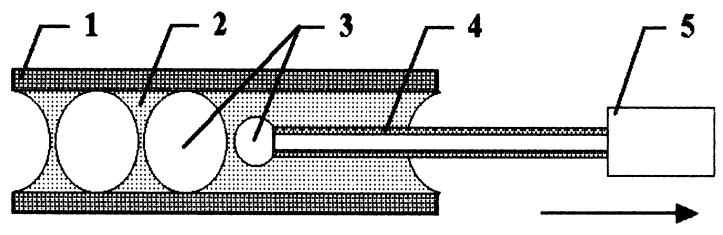

Fig. 2. Schematic view of the setup for the fabrication of the microcapillary lens. 1 - glass capillary tube; 2 - glycerine; 3 - air bubbles; 4 - long glass microcapillary injector needle; 5 - cylinder with a compressed air.

lary- 1 and its outer diameter is significantly smaller than the inner one of capillary-1. This "glass needle" is produced by the gravity puller method described in Ref. [11]. The "needle" is connected to the cylinder with compressed air and is inserted into capillary-1. Then the air is injected through capillary-2 into capillary- 1 . The process of the air bubble being formed is observed under the microscope. When the air bubbles with the diameters of capillary-1 are formed, the "needle" is moved so that the distance between the air bubble and the "needle" is several microns. By repeating this procedure, a sufficiently large number of bubbles are injected into capillary-1. The liquid between the neighboring bubbles has an approximately spherical concave shape.

Glycerin was used for the lens. We have found that the method allows us to produce glycerine lenses of $10-20 \mu \mathrm{m}$ in thickness $d$ inside the glass capillaries with the diameter of $0.1-0.2 \mathrm{~mm}$. For the capillaries with the diameters of $0.3-0.8 \mathrm{~mm}$, the measured lens thickness ranged from 30 to $150 \mu \mathrm{m}$. A visible-light microscope image of the capillary $\mathrm{X}$-ray lenses is shown in Fig. 3. The air bubbles appear as light circles. The lenses do not seem spherical due to the refraction of light at the air/glass boundary. The diameter of the capillary is equal to $0.8 \mathrm{~mm}$.

\section{Ray tracing simulations}

The properties of the compound X-ray lens (focal length, spot size, gain and so on) depend on the surface shape of the individual lens, the number of 
the lenses and the lens thickness $d$. These data are assumed for ray-tracing calculations.

The main features of the ray-tracing simulations are the following. The X-ray source is treated as a point source with an isotropic emission of the photons in all directions. In simulations a large number of ray paths are generated from the source.

Ray tracing calculations are made for the microcapillary lens used in experiments for focusing of $18.3 \mathrm{keV}$ X-rays at SPring-8 [10]. The lens was prepared by method 1 described in Section 2 . A commercial epoxy glue was used for the lens fabrication. The density of the used epoxy glue is equal to $1.08 \mathrm{~g} \mathrm{~cm}^{3}$, and the chemical formula is estimated to be $\mathrm{C}_{20} \mathrm{H}_{30} \mathrm{O}_{2}$. The radius of the used capillary is $R=0.104 \mathrm{~mm}$, and the number of the microlenses $N=71$. The microlens thickness $d=0.25 \mathrm{~mm}$ was calculated from Eq. (2). The distances between the lenses are assumed to have the same value of $0.45 \mathrm{~mm}$. The shape of the lens surface was approximated by a sphere with the inner radius of the capillary. The expected linear absorption coefficient for the utilized epoxy was $0.55 \mathrm{~cm}^{-1}$ at $18 \mathrm{keV}$. The refractive index $n$ for $\mathrm{X}$-rays is given as [1]

$n=1-\delta-\mathrm{i} \beta$

where $\delta$ is the refractive index decrement and $\beta$ is related to the absorption of X-rays. For the X-ray photons, with energies far from absorption edges of the materials inside the lens, the decrement $\delta$ can be simply approximated by

$\delta=N_{\mathrm{a}} /(2 \pi) r_{0} \lambda^{2} \rho Z / A$

where $N_{\mathrm{a}}$ is the Avogadro's number, $r_{0}$ is the classical electron radius, $\rho$ is the density, $\lambda$ is the $\mathrm{X}$-ray wavelength, and $Z$ and $A$ are the atomic number and mass, respectively. For the used epoxy, $\delta=7.4 \times 10^{-7}$ for $18.3 \mathrm{keV}$ X-rays.

Fig. 4 shows the paths of the X-rays around the focal spot. The incident X-ray beam was generated by a point source located at infinity $(500 \mathrm{~m}$ to the lens). Experimentally [10], the X-ray source is located at a distance of $45 \mathrm{~m}$ from the lens. For this case the paths of X-rays are very close to the ones shown in Fig. 4. With reference to Fig. 4, it can be seen that the focal length of the lens is about $1 \mathrm{~m}$. This result is in reasonably good agreement with
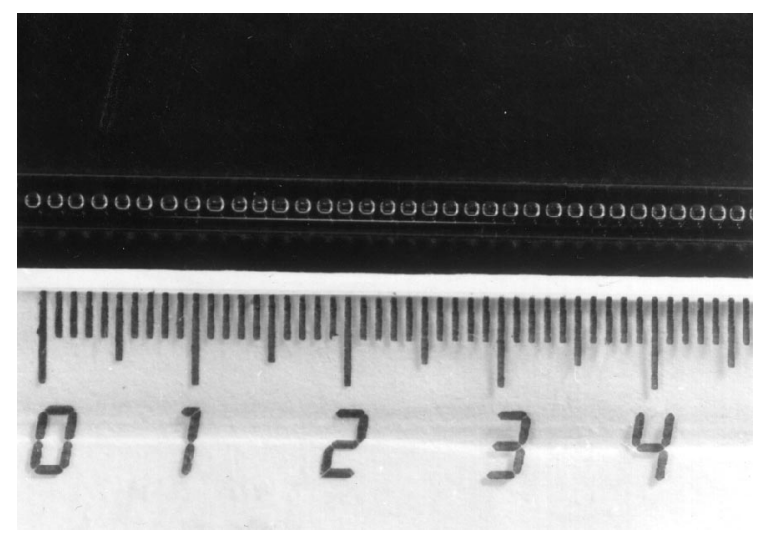

Fig. 3. Visible light microscope images of the capillary X-ray lens. The individual lenses are made from glycerine. The air bubbles between lenses appear as light circles. The diameter of the capillary is equal to $0.8 \mathrm{~mm}$.

the experimentally deduced focal position that was observed somewhere between 0.8 and $0.9 \mathrm{~m}$ [10].

The X-ray intensity distribution $F(r, z)$ is calculated, where $r$ and $z$ represents the distance of the detector from the optical axis and the distance from the lens to the detector, respectively. The intensity distributions at different $z$ are shown in Fig. 5. The profiles in Fig. 5 are normalized by the following equation:

$2 /\left(R^{2} T\right) \int F(r, z) r \mathrm{~d} r=\varphi$

where $R$ is the radius of the lens, $\varphi$ is the X-ray flux density at the entrance of the lens, $T$ is the lens transparency. The $F(r, z)$-distributions shown in Fig. 5 are pure geometric optics calculations. These calculations are not valid for a focus size close to the diffraction limit (e.g. $0.4 \mu \mathrm{m}$ for the X-ray energy $E=18.3 \mathrm{keV}$, radius of the microlens $R=0.104 \mathrm{~mm}$, and the focal length $F=1 \mathrm{~m}$ ).

As the compound lens is an array of spherical lenses, spherical aberrations occur just in the same way as for spherical visual-light lens. Three planes around the focus may be denoted to describe the lens aberrations. They are shown by the lines of MP, MS and PP in Fig. 4. The plane PP represents a focal plane. The plane MS represents the circle of the least confusion [12]. The marginal rays focus in 


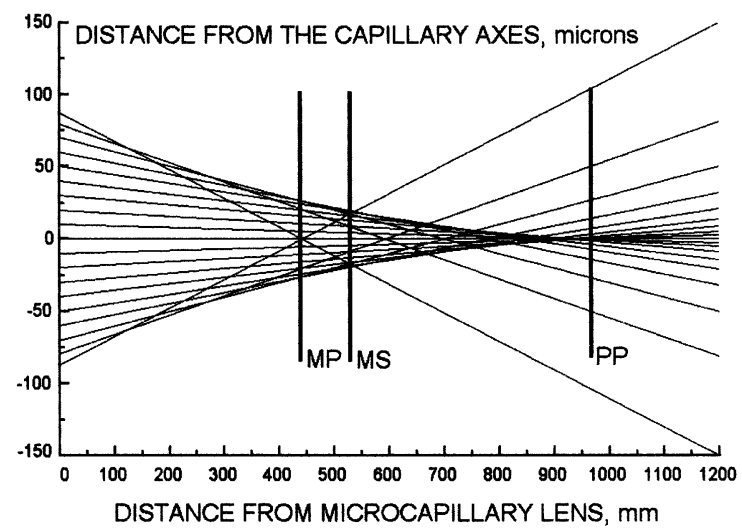

Fig. 4. Paths of the X-rays forming a focal spot of the microcapillary lens. The photons energy, the inner radius of the capillary and the number of lenses are assumed to be $18.3 \mathrm{keV}$, $0.104 \mathrm{~mm}$ and 71 .

the plane MP. The beam radius $R_{\mathrm{pp}}$ at the PP plane is calculated from the third-order aberration theory [12] as the following equation assuming a point source located at the infinity and spherical lenses:

$R_{\mathrm{pp}}=F B R_{\mathrm{d}}^{3}$

and

$$
\begin{aligned}
B= & \left\{1 / R_{1}^{3}(n-1) / n^{2}+\left[n / R_{2}-(n-1) / R_{1}\right]^{2}\right. \\
& \left.\times\left[(1-n) / R_{2}+(n-1) / R_{1}-(n-1) / n^{2} / R^{1}\right]\right\} / 2
\end{aligned}
$$

where $F$ is the focal length of the lens, $B$ is the Seidel coefficient, $R_{\mathrm{d}}$ is the radius of the diaphragm placed before the lens, $n$ is the refractive index and $R_{1}$ and $R_{2}$ are the radii of curvature of the lenses at two surfaces. The relation between the beam radius at the PP-plane $\left(R_{\mathrm{pp}}\right)$ and at the MS plane $\left(R_{\mathrm{ms}}\right)$ is [13]

$R_{\mathrm{ms}}=\frac{1}{4} R_{\mathrm{pp}}$.

The distance $L_{\mathrm{ms}}$ between the MS-plane and the lens satisfies the following equation:

$L_{\mathrm{ms}}=F\left(R_{\mathrm{d}}+R_{\mathrm{ms}}\right) /\left(R_{\mathrm{d}}+R_{\mathrm{pp}}\right)$.

The focal length of the equi-concave lens $\left(-R_{1}=R_{2}=R\right)$ can be written in the conventional form

$F=-R / 2(n-1)$.
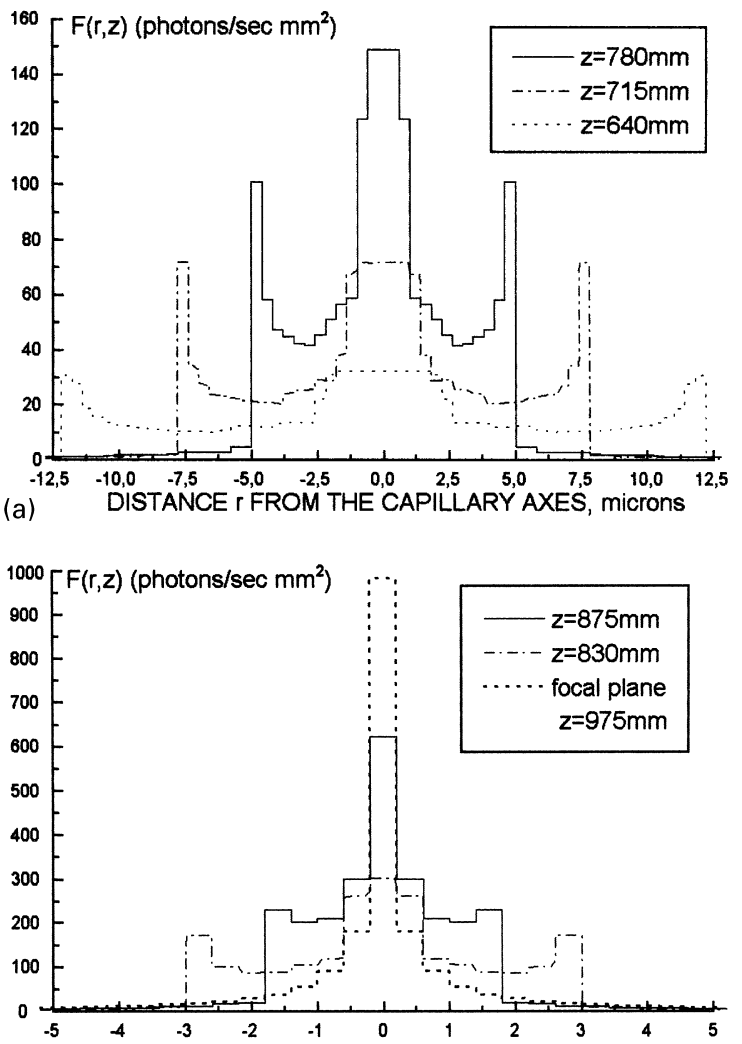

(b) DISTANCE r FROM THE CAPILLARY AXES, microns

Fig. 5. Ray tracing calculations for the intensity distribution function $F(r, z)$ for $18.3 \mathrm{keV}$ photons at (a) the distances $z=640 \mathrm{~mm}, 715$ and $780 \mathrm{~mm}$, and (b) at the distances $z=830$, 875 and $975 \mathrm{~mm}$ (PP plane) from the microcapillary lens. The assumed lens parameters are $R=0.104 \mathrm{~mm}, N=71, F=1 \mathrm{~m}$. $F(r, z)$ function is normalized in accordance with Eq. (5) for $T=0.3$ and $\varphi=1$ photon $/\left(\mathrm{s} \mathrm{mm}^{2}\right)$.

The focal length of the compound X-ray lens is defined as [2]

$F=R / 2 \delta N$.

When this result is compared with that of Eq. (10), it is apparent that the X-rays very close to the optical axis are focused by the compound lens in the same way as by the lens with refractive index $n$ calculated as $n=1-\delta N$. Using these formulas for $R_{\mathrm{pp}}, R_{\mathrm{ms}}$, the following equations for the spherical bi-concave X-ray lens may be obtained:

$R_{\mathrm{pp}}=\left(\frac{1}{2}\right) R_{\mathrm{d}}^{3} / R^{2}$ 


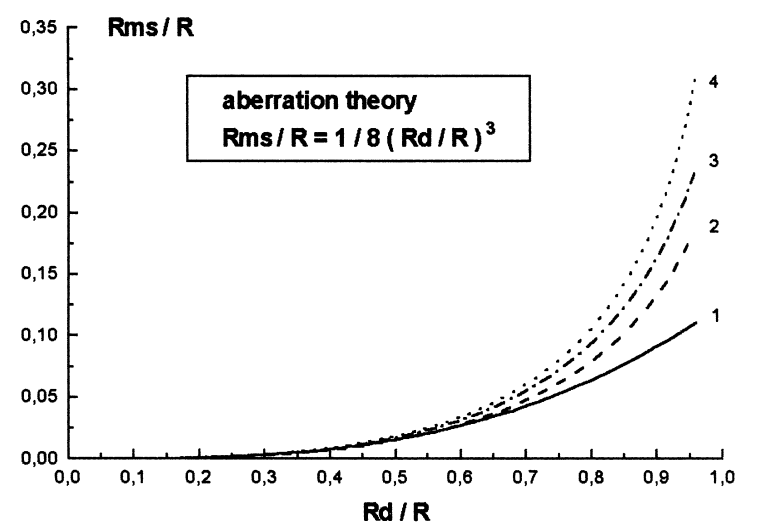

Fig. 6. Dependence of $R_{\mathrm{ms}} / R$ on $R_{\mathrm{d}} / R$ based on the third-order aberration theory and ray tracing calculations for $18.3 \mathrm{keV} \mathrm{X}$ rays. Line 1 - aberration theory; line 2 - ray tracing calculations (RTC) for the lens with $R=0.5 \mathrm{~mm}, N=340, F=1 \mathrm{~m}$; line 3 - RTC for the lens with $R=0.3 \mathrm{~mm}, N=204, F=1 \mathrm{~m}$; line 4 - RTC for the lens with $R=0.1 \mathrm{~mm}, N=68, F=1 \mathrm{~m}$. The X-ray source is located at infinity.

$R_{\mathrm{ms}}=\left(\frac{1}{8}\right) R_{\mathrm{d}}^{3} / R^{2}$.

The analytical results for $F$ and $R_{\mathrm{ms}}$ values were compared with the ray tracing calculations. The theoretical focal length shown by Eq. (11) is $F=995 \mathrm{~mm}$ for $18.3 \mathrm{keV} \mathrm{X}$-rays. This is in good agreement with the focal length obtained by ray tracing calculations which was equal to $F=975 \mathrm{~mm}$. As for $R_{\mathrm{ms}}$, Fig. 6 displays the dependence of $R_{\mathrm{ms}} / R$ on $R_{\mathrm{d}} / R$ for the theoretical case (curved line 1) and for the ray-tracing calculations (curved lines 2-4). A good agreement is seen between the data under the condition $R_{\mathrm{d}} / R$ is less than 0.6. In other words, the replacement of $n=1-\delta$ by $n=1-\delta N$ seems reasonable with the assumptions of (i) a point source located at infinity, (ii) the lens length is significantly less than the lens focal length and (iii) the diaphragm sets upstream of the lens with the radius being less than $0.6 R$, where $R$ is the lens radius.

\section{Discussions on the simulations}

The performance of the X-ray lens may be described by the gain $G$ in intensity of the X-ray beam at the lens focal plane compared with the intensity which would have been obtained without a lens. For a spherical lens the MS plane is every bit as important as the focal plane and the gain may be defined as

$G=\varphi_{1} / \varphi$

where $\varphi$ and $\varphi_{1}$ are the flux densities of the X-rays before the lens and at the PP (or at the MS) plane, respectively. For a finite-sized sample the gain depends on the sample size. For example, we consider a case when the sample is located in the focal plane of the lens and no diaphragm is used. In this case the gain $G$ may be calculated as

$G=R_{1}^{2} / R_{\mathrm{s}}^{2} T$

where $R_{\mathrm{s}}, T$ and $R_{1}$ are the radius of the sample, the $\mathrm{X}$-ray transmissivity of the lens and the radius of the X-ray beam forming the focal spot of $R_{\mathrm{s}}$ in radius, respectively. The $R_{1}$ is obtained from Eq. (12) $\left(R_{\mathrm{pp}}=R_{\mathrm{s}}\right)$ as

$R_{1}=\left(2 R_{\mathrm{s}} R^{2}\right)^{1 / 3}$.

The maximum gain $G_{\max }$ is obtained when the sample radius $R_{\mathrm{s}}$ equals $R_{\text {diff }}$, where $R_{\text {diff }}$ is a diffraction-limited radius which is approximated by the radius of the first minimum of the Airy diffraction pattern in the focal plane [1]:

$R_{\text {diff }}=0.61 \lambda F / R$

where $\lambda$ is the wavelength of the $X$-radiation. As an example, we consider a case of the microcapillary lens used in Ref. [10] for focusing $18 \mathrm{keV}$ X-rays (with lens radius $R=0.104 \mathrm{~mm}, N=71$, focal length $F=1 \mathrm{~m}$ for $18 \mathrm{keV} \mathrm{X-rays).} \mathrm{The} \mathrm{calculated}$ values of $R_{\text {diff }}$ and $R_{1}$ are $R_{\text {diff }}=0.4 \mu \mathrm{m}$, $R_{1}=21 \mu \mathrm{m}$. Transmissivity $T$ can be approximated by $T=\exp (-\mu \mathrm{Nd})$, where $d$ is the individual lens thickness, $\mu$ is the linear absorption coefficient for $18 \mathrm{keV}$ X-rays. Assuming $d=0.25 \mathrm{~mm}$ after Eq. (2), $T=0.38$ for $18 \mathrm{keV} \mathrm{X-}$ rays is obtained and the gain $G_{\max }$ equals 1000 .

As can be seen from Fig. 4 the large aberrations of the microcapillary lens need to be reduced for some experiments (e.g. for a scanning X-ray microscope where a small beam of $1 \mu \mathrm{m}$ in diameter is needed). These aberrations can be reduced by placing a diaphragm of $R_{\mathrm{d}}$ in radius before the lens and putting the sample in the position of MS-plane. The 
diaphragm is used for reducing the radius of the "circle of least confusion" $R_{\mathrm{ms}}$ up to the value of $R_{\text {ms-min }}$. The optimal radius of the diaphragm $R_{\mathrm{d}-\mathrm{opt}}$ and the minimum radius of the "circle of least confusion" $R_{\mathrm{ms}-\mathrm{min}}$ in this case is shown in the following equations:

$R_{\mathrm{ms}-\mathrm{min}}=\frac{1}{8} R_{\mathrm{d}-\mathrm{opt}}^{3} / R^{2}$
$R_{\mathrm{ms}-\min }=0.61 \lambda L_{\mathrm{ms}} / R_{\mathrm{d}-\mathrm{opt}}$

where $L_{\mathrm{ms}}$ is the distance between the lens and the MS-plane. The solution of Eqs. (18) for $R_{\mathrm{d} \text {-opt }}$ and $R_{\mathrm{ms}-\mathrm{min}}$ under the assumption $L_{\mathrm{ms}}=F$ is

$R_{\mathrm{d}-\mathrm{opt}}=1.49(\lambda F)^{1 / 4} R^{1 / 2}$.

$R_{\mathrm{ms}-\min }=0.41(\lambda F)^{3 / 4} / R^{1 / 2}$

and the gain $G$ for this case is

$G=\left(R_{\mathrm{d}-\mathrm{opt}} / R_{\mathrm{ms}-\mathrm{min}}\right)^{2} T$

where $T$ is the X-ray transmissivity of the lens.

For example, a diaphragm is set upstream of the above-mentioned lens and $18 \mathrm{keV} \mathrm{X}$-rays is assumed to be used. The optimum radius of the diaphragm $R_{\mathrm{d}-\mathrm{opt}}$ (for achieving $R_{\mathrm{ms}}=R_{\mathrm{ms}-\mathrm{min}}$ ) in this case is $R_{\mathrm{d}-\mathrm{opt}}=44 \mu \mathrm{m}$ and $R_{\mathrm{ms}-\min }=0.96 \mu \mathrm{m}$. Assuming $T=0.38$ for $18 \mathrm{keV} \mathrm{X-rays,} \mathrm{the} \mathrm{gain}$ $G=798$.

Eq. (20) shows that $R_{\mathrm{ms}-\mathrm{min}}$ decreases as the lens radius $R$ increases, and $R_{\mathrm{ms}-\min }=0.5 \mu \mathrm{m}$ can be achieved for a lens $(R=0.384 \mathrm{~mm}$, for $18 \mathrm{keV} \mathrm{X}$ rays and $F=1 \mathrm{~m}) \cdot R_{\mathrm{d} \text {-opt }}=0.084 \mathrm{~mm}$ and $N=252$ lenses are needed in this case and the expected gain in the MS-plane is $G=28224 \mathrm{~T}$. The lenses with the thickness of $0.05-0.2 \mathrm{~mm}$ can be fabricated by method 2 described in Section 2. The expected value of $T$ for a lens with 252 microlenses with the thickness of $0.05-0.2 \mathrm{~mm}$ is $T=0.5-0.06$ and the expected value of $G=14000-1600$.

The calculations have shown a possibility to obtain a pronounced focusing effect (gain about 1000 at $18 \mathrm{keV}$ for an ideal case of a point source) by using a microcapillary lens with an inner radius of $100 \mu \mathrm{m}$. The conditions of the experiment [10] differ from the ideal ones: the undulator source size in FWHM was expected to be $800 \mu \mathrm{m} \times 30 \mu \mathrm{m}$ and the source is located at the distance $L=45 \mathrm{~m}$ to the lens. For this case the expected size of the X-ray spot at the focal plane is $F /(L-F)$ times less than the source size, where $F$ is the focal length. There are some problems to calculate gain $G_{\exp }$ for the conditions of the experiment [10], but one would expect that it is little more than $G_{\exp }$ which is equal to

$G_{\exp }=\pi R_{1}^{2} /\left(S_{x} S_{y}\right) T$

where the value $R_{1}=21 \mu \mathrm{m}$ is calculated after Eq. (16) under the assumption $R_{\mathrm{s}}=R_{\text {diff }}$ and $S_{x} S_{y}=18 \mu \mathrm{m} \times 0.7 \mu \mathrm{m}$ is the expected X-ray spot size. The gain obtained in the experiment [10] was about 12 and is a few times smaller than the expected gain $G_{\text {exp }}=42$. This disagreement may be attributed to the diffuse $X$-ray scattering and the small-angle scattering of X-rays in the lens material which resulted in blurring of the focus spot as was observed in Ref. [10].

It is evident that these phenomena play a less significant role for the microlenses produced by the second method as described in Section 2. Furthermore, the method is best suited for fabricating $\mathrm{X}$-ray lens with focal length up to $10-20 \mathrm{~cm}$ for $18 \mathrm{keV}$ X-rays. The lens may be useful for demagnification of the X-ray source size up to some microns and we plan to consider this case in a comparison publication.

\section{References}

[1] A.G. Michette, Optical Systems for Soft X-rays, Plenum Press, New York, 1986.

[2] A. Snigirev, V. Kohn, I. Snigireva, B. Lengeler, Nature 384 (1996) 49.

[3] A. Snigirev, V. Kohn, I. Snigireva, A. Souvorov, B. Lengeler, Appl. Opt. 37 (1998) 653.

[4] P. Elleaume, J. Synchrotron Radiat. 5 (1998) 1.

[5] B. Lengeler, J. Tummler, A. Snigirev, I. Snigireva, C. Raven, J. Appl. Phys. 84 (1998) 5855.

[6] B. Lengeler, C.G. Schroer, M. Richwin, J. Tummler, M. Drakopoulos, A. Snigirev, I. Snigireva, Appl. Phys. Lett. 74 (1999) 3924.

[7] Y. Kohmura, K. Sato, Y. Ishisawa, Y. Suzuki, T. Ishikawa, SPring-8 Annual Report, 1997, p. 239.

[8] Y. Kohmura, M. Awaji, Y. Suzuki, T. Ishikawa, Proc. SPIE 3449 (1998) 185.

[9] Yu.I. Dudchik, N.N. Kolchevsky, Nucl. Instr. and Meth. A 421 (1999) 361.

[10] Y. Kohmura, M. Awaji, Y. Suzuki, T. Ishikawa, Yu.I. Dudchik, N.N. Kolchevsky, F.F. Komarov, Rev. Sci. Instr. 70 (1999) 4161. 
[11] D.J. Thiel, D.H. Bilderback, A. Lewis, Rev. Sci. Instr. 64 (1993) 2873.

[12] M. Born, E. Wolf, Principles of Optics, 5th edition, Pergamon Press, Elmsford, New York, 1975.
[13] P.W. Hawkes, E. Kasper, Principles of Electron Optics, Vol. 1, Academic Press, New York, 1989. 